
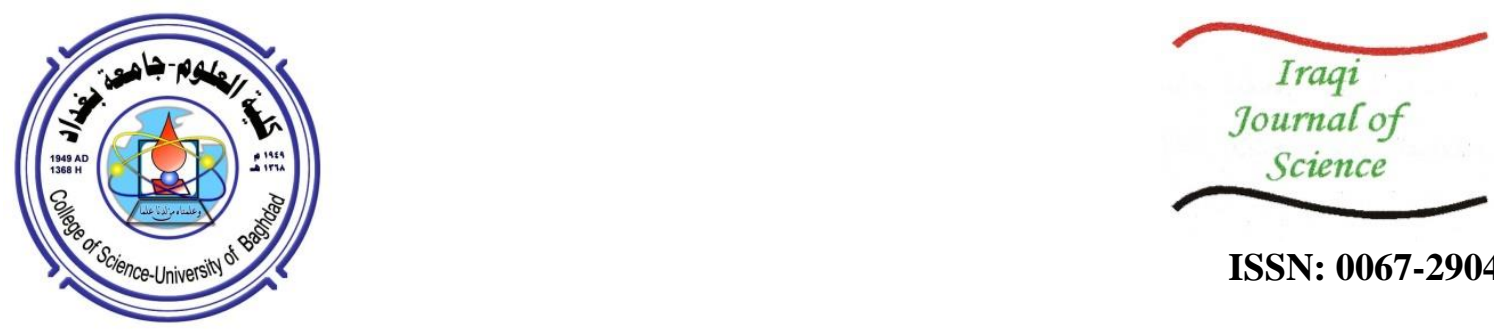

ISSN: 0067-2904

\title{
Soft Continuous Mappings in Soft Closure Spaces
}

\author{
S. T. Ekram, ${ }^{1}$ R. N. Majeed \\ University of Baghdad, Faculty of Education for Pure Sciences Abn Al-Haitham, Department of \\ Mathematics,Baghdad, Iraq
}

Received: 5/8/2020 Accepted: 9/11/2020

\begin{abstract}
Soft closure spaces are a new structure that was introduced very recently. These new spaces are based on the notion of soft closure operators. This work aims to provide applications of soft closure operators. We introduce the concept of soft continuous mappings and soft closed (resp. open) mappings, support them with examples, and investigate some of their properties.
\end{abstract}

Keywords: Soft closure operator, soft closure space, closed soft set, product of soft closure spaces, soft continuous mapping, soft closed mapping, soft projection mapping.

\section{التطبيقات المستمرة الناعمة في فضاءات الاغلاق الناعمة}

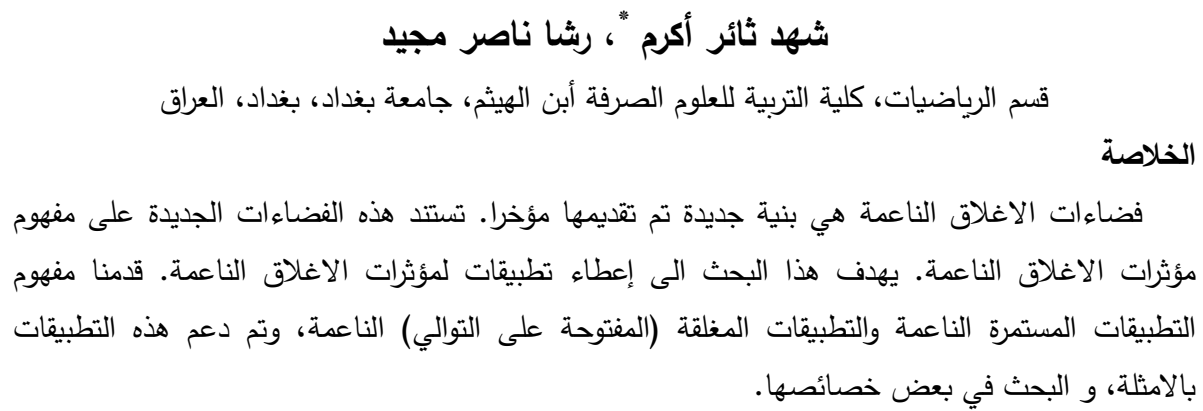

\section{Introduction}

The concept of soft sets was first introduced by Molodtsov [1] in 1999 as a general mathematical tool for dealing with uncertain objects. Soft set theory has been applied in many directions, e.g., stability and regularization [1], game theory and operations research [1], soft analysis [1], group theory [2], general topology [3], etc. Moreover, in the structure of closure spaces, Gowri and Jegadeesan [4] and Krishnaveni and Sekar [5] introduced and studied $\breve{C}$ ech soft closure spaces. In the classical soft set theory, because of the fuzzy existence of the parameters, a condition can be complicated in the real world. In this respect, classical $\breve{C}$ ech soft closure spaces were expanded to $\breve{C}$ ech soft closure spaces $[6,7,8]$. Recently, Ekram and Majeed introduced the notion of soft closure spaces [9] as an expansion to this concept in the ordinary case of the set theory that was introduced by Čech [10].

Continuity is an important notion in general topology, soft topology, and closure spaces as well as all branches of mathematics and quantum physics. Kharal and Ahmad [11] presented the concept of a mapping on the classes of soft sets that is a central notion for the advancement of every new field of mathematical science. An idea of soft mapping was presented and some of its properties were studied 
in [12]. Boonpok [13] defined and studied the concept of continuity in closure spaces. Our work in the present paper is dedicated to presenting the concept of soft continuity in soft closure spaces. In Section 3 , we introduce the concept of soft continuous and study some of their properties. Also, the notion of soft closed (resp. open) mappings is introduced. In Section 4, several properties and characterizations related to soft projection mappings, closed (resp. open) soft sets, and soft continuous (resp. soft closed) mappings in the product of soft closure spaces are discussed.

\section{Preliminaries}

In this section, we introduce the basic definitions and results of soft set theory and soft closure spaces that will be needed in the sequel.

Definition 2.1[1]. A soft set $\mathcal{F}_{R}=(\mathcal{F}, R)$ over the universe set $\mathcal{M}$ is defined by a mapping $\mathcal{F}: R \rightarrow$ $P(\mathcal{M})$. Then, $\mathcal{F}_{R}$ can be represented by the set $\mathcal{F}_{R}=\{(r, \mathcal{F}(r)): r \in R$ and $\mathcal{F}(r) \in P(\mathcal{M})\}$. We denote the family of all soft sets over $\mathcal{M}$ by $\mathcal{S} \mathcal{S}(\mathcal{M}, R)$.

Definition 2.2 [14]. A null soft set, denoted by $\widetilde{\Phi}_{R}$, is a soft set $\mathcal{F}_{R}$ over $\mathcal{M}$ such that for all $r \in R$, $\mathcal{F}(R)=\emptyset$ (empty set).

Definition 2.3 [14]. An absolute soft set, denoted by $\tilde{\mathcal{M}}$, is a soft set $\mathcal{F}_{R}$ over $\mathcal{M}$ such that for all $r \in R, \mathcal{F}(r)=\mathcal{M}$.

Definition 2.4 [15]. Let $\mathcal{F}_{R}$ and $G_{R}$ be two soft sets over $\mathcal{M}$. Then, $\mathcal{F}_{R}$ is called a soft subset of $G_{R}$, denoted by $\mathcal{F}_{R} \subseteq G_{R}$, if $\mathcal{F}(r) \subseteq G(r)$ for all $r \in R$. $\mathcal{F}_{R}$ equals $G_{R}$, denoted by $\mathcal{F}_{R}=G_{R}$, if $\mathcal{F}_{R} \subseteq G_{R}$ and $G_{R} \sqsubseteq \mathcal{F}_{R}$.

Definition 2.5 [14]. The union of two soft sets $\mathcal{F}_{R}$ and $G_{R}$ over $\mathcal{M}$ is the soft set $\mathcal{H}_{R}$ defined as b $\mathcal{H}(r)=\mathcal{F}(r) \cup G(r)$ for all $r \in R$. This is denoted by $\mathcal{F}_{R} \sqcup G_{R}$. Also, the soft intersection of $\mathcal{F}_{R}$ and $G_{R}$ is the soft set $\mathcal{H}_{R}$ given by $\mathcal{H}(r)=\mathcal{F}(r) \cap G(r)$ for all $r \in R$ and denoted by $\mathcal{F}_{R} \sqcap G_{R}$.

Definition 2.6 [3]. Let $\mathcal{F}_{R}$ and $G_{R}$ be two soft sets over $\mathcal{M}$, the difference $\mathcal{H}_{R}$ of $\mathcal{F}_{R}$ and $G_{R}$ is denoted by $\mathcal{F}_{R}-G_{R}$, and defined as $\mathcal{H}(r)=\mathcal{F}(r)-G(r)$ for all $r \in R$.

Definition 2.7 [3]. The relative complement of a soft set $\mathcal{F}_{R}$ is denoted by $\mathcal{F}_{R}^{c}$, where $\mathcal{F}^{c}: R \rightarrow P(\mathcal{M})$ defined as $\mathcal{F}^{c}(r)=\mathcal{M}-\mathcal{F}(r)$, for all $r \in R$. Clearly, $\mathcal{F}_{R}^{c}=\widetilde{\mathcal{M}}-\mathcal{F}_{R}$.

Definition 2.8 [16]. The soft set $\mathcal{F}_{R} \in \mathcal{S} \mathcal{S}(\mathcal{M}, R)$ is called soft point in $\mathcal{M}$, denoted by $x_{r}$, if for the element $r \in R$, we have $\mathcal{F}(r)=\{x\}$ and $\mathcal{F}\left(r^{\prime}\right)=\emptyset$ for every $r^{\prime} \in R-\{r\}$.

Definition 2.9 [16]. The soft point $x_{r}$ is said to be in the soft set $G_{R}$, denoted by $x_{r} \widetilde{\in} G_{R}$, if for the element $r \in R$, we have $\{x\} \subseteq G(r)$.

Definition 2.10 [11]. Let $\mathcal{S} \mathcal{S}(\mathcal{M}, R)$ and $\mathcal{S} \mathcal{S}(\mathcal{N}, \mathcal{K})$ be families of soft sets, where $\psi: \mathcal{M} \rightarrow \mathcal{N}$ and $\ell: R \rightarrow \mathcal{K}$ are mappings. The mapping $\psi_{\ell}: \mathcal{S} \mathcal{S}(\mathcal{M}, R) \rightarrow \mathcal{S} \mathcal{S}(\mathcal{N}, \mathcal{K})$ is defined as:

1- $\quad$ If $\mathcal{F}_{R} \in \mathcal{S} \mathcal{S}(\mathcal{M}, R)$, then the image of $\mathcal{F}_{R}$ under $\psi_{\ell}$, written as $\psi_{\ell}\left(\mathcal{F}_{R}\right)=\left(\psi_{\ell}\left(\mathcal{F}_{R}\right), \ell(R)\right)$, is a soft set in $\mathcal{S} \mathcal{S}(\mathcal{N}, \mathcal{K})$ such that:

$$
\psi_{\ell}\left(\mathcal{F}_{R}\right)(k)=\left\{\begin{array}{cl}
\psi\left(\cup_{r \in \ell_{(k) \cap R}^{-1}} \mathcal{F}(r)\right), & \text { if } r \in \ell^{-1}(k) \cap R \neq \emptyset ; \\
\emptyset, & \text { otherwise. }
\end{array}\right.
$$

2- If $G_{\mathcal{K}} \in \mathcal{S} \mathcal{S}(\mathcal{N}, \mathcal{K})$, then the pre-image of $G_{\mathcal{K}}$ under $\psi_{\ell}$, written as $\psi_{\ell}^{-1}\left(G_{\mathcal{K}}\right)=$ $\left(\psi_{\ell}{ }^{-1}\left(G_{\mathcal{K}}\right), \ell^{-1}(\mathcal{K})\right)$, is a soft set in $\mathcal{S} \mathcal{S}(\mathcal{M}, R)$ such that

$$
\psi_{\ell}^{-1}\left(G_{\mathcal{K}}\right)(r)=\left\{\begin{array}{cc}
\psi^{-1}(G(\ell(r))), & \text { if } \ell(r) \in \mathcal{K} ; \\
\varnothing, & \text { otherwise. }
\end{array}\right.
$$

Definition 2.11 [17]. Let $\mathcal{F}_{R} \in \mathcal{S} \mathcal{S}(\mathcal{M}, R)$ and $G_{\mathcal{K}} \in \mathcal{S} \mathcal{S}(\mathcal{N}, \mathcal{K})$. The Cartesian product $\mathcal{F}_{R} \times G_{\mathcal{K}}$ is defined by $(\mathcal{F} \times G)_{R \times \mathcal{K}}$ where

$(\mathcal{F} \times G)_{R \times \mathcal{K}}(r, k)=\mathcal{F}(r) \times G(k)$, for all $(r, k) \in R \times \mathcal{K}$.

From this definition, the soft set $\mathcal{F}_{R} \times G_{\mathcal{K}}$ is a soft set over $\mathcal{M} \times \mathcal{N}$ and its universe parameter is $R \times \mathcal{K}$.

The pairs of projections $p_{\mathcal{M}}: \mathcal{M} \times \mathcal{N} \rightarrow \mathcal{M}, q_{R}: R \times \mathcal{K} \rightarrow R$ and $p_{\mathcal{N}}: \mathcal{M} \times \mathcal{N} \rightarrow \mathcal{N}, q_{S}: R \times \mathcal{K} \rightarrow$ $\mathcal{K}$ determine, respectively, the morphisms $\left(p_{\mathcal{M}}, q_{R}\right)$ from $\mathcal{M} \times \mathcal{N}$ to $\mathcal{M}$ and $\left(p_{\mathcal{N}}, q_{\mathcal{K}}\right)$ from $\mathcal{M} \times \mathcal{N}$ to $\mathcal{N}$, where

$\left(p_{\mathcal{M}}, q_{R}\right)\left(\mathcal{F}_{R} \times G_{\mathcal{K}}\right)=p_{\mathcal{M}}(\mathcal{F} \times G)_{q_{R}(R \times \mathcal{K})}$ and

$\left(p_{\mathcal{N}}, q_{\mathcal{K}}\right)\left(\mathcal{F}_{R} \times G_{\mathcal{K}}\right)=p_{\mathcal{N}}(\mathcal{F} \times G)_{q_{S}(R \times \mathcal{K})}[18]$. 
Definition 2.12 [9]. An operator $\tilde{u}: \mathcal{S} \mathcal{S}(\mathcal{M}, R) \rightarrow \mathcal{S} \mathcal{S}(\mathcal{M}, R)$ is called a soft closure operator (soft- $c o$, for short) on $\mathcal{M}$, if for all $\mathcal{F}_{R}, G_{R} \in \mathcal{S} \mathcal{S}(\mathcal{M}, R)$ the following axioms are satisfied:

(C1) $\widetilde{\Phi}_{R}=\tilde{u}\left(\widetilde{\Phi}_{R}\right)$;

$(\mathcal{C} 2) \mathcal{F}_{R} \sqsubseteq \tilde{u}\left(\mathcal{F}_{R}\right)$;

(C3) $\mathcal{F}_{R} \sqsubseteq G_{R} \Longrightarrow \tilde{u}\left(\mathcal{F}_{R}\right) \sqsubseteq \tilde{u}\left(G_{R}\right)$.

The triple $(\mathcal{M}, \tilde{u}, R)$ is called a soft closure space (soft-cs, for short). A soft subset $\mathcal{F}_{R}$ over $\mathcal{M}$ is said to be a closed soft set, if $\mathcal{F}_{R}=\tilde{u}\left(\mathcal{F}_{R}\right)$. A soft subset $G_{R}$ over $\mathcal{M}$ is called an open soft set if it is relative complement $\widetilde{\mathcal{M}}-\mathcal{F}_{R}$ and is a closed soft set.

Definition 2.13 [9]. Let $(\mathcal{M}, \tilde{u}, R)$ be a soft-cs and let $\mathcal{Y} \subseteq \mathcal{M}$. Let $\tilde{u}_{y}: \mathcal{S} \mathcal{S}(\mathcal{Y}, R) \rightarrow \mathcal{S} \mathcal{S}(\mathcal{Y}, R)$ defined by $\tilde{u}_{y}\left(\mathcal{F}_{R}\right)=\tilde{\mathcal{Y}} \sqcap \tilde{u}\left(\mathcal{F}_{R}\right)$. Then, $\tilde{u}_{y}$ is called the relative soft closure operator on $\mathcal{Y}$ induced by $\tilde{u}$. The triple $\left(\mathcal{Y}, \tilde{u}_{y}, R\right)$ is called a soft closure subspace (soft-c.subsp, for short) of $(\mathcal{M}, \tilde{u}, R)$.

Theorem 2.14 [9]. Let $\left\{\left(\mathcal{M}_{\alpha}, \tilde{u}_{\alpha}, R_{\alpha}\right): \alpha \in \mathcal{J}\right\}$ be a family of soft-cs's. If $G_{\prod_{\alpha \in \mathcal{J}} R_{\alpha}}$ is an open soft set in the product soft-cS $\left(\prod_{\alpha \in \mathcal{J}} \mathcal{M} \mathcal{M}_{\alpha}, \otimes \tilde{u}, \prod_{\alpha \in \mathcal{J}} R_{\alpha}\right)$, then $\left(p_{\mathcal{M}_{\alpha}}, q_{R_{\alpha}}\right)\left(G_{\prod_{\alpha \in \mathcal{J}} R_{\alpha}}\right)$ is an open soft set in $\left(\mathcal{M}_{\alpha}, \tilde{u}_{\alpha}, R_{\alpha}\right)$ for all $\alpha \in \mathcal{J}$.

\section{Soft continuous mappings}

In this section, we introduce the concept of soft continuous (resp. soft closed) mappings between soft closure spaces, with some examples to explain these notions. Also, some properties related to these concepts are given.

Definition 3.1. Let $(\mathcal{M}, \tilde{u}, R)$ and $(\mathcal{N}, \tilde{v}, \mathcal{K})$ be soft-cs's. A soft mapping $\psi_{\ell}:(\mathcal{M}, \tilde{u}, R) \rightarrow(\mathcal{N}, \tilde{v}, \mathcal{K})$ is said to be soft continuous, if $\psi_{\ell}\left(\tilde{u}\left(\mathcal{F}_{R}\right)\right) \sqsubseteq \tilde{v}\left(\psi_{\ell}\left(\mathcal{F}_{R}\right)\right)$ for every soft set $\mathcal{F}_{R} \in \mathcal{S} \mathcal{S}(\mathcal{M}, R)$.

In the next paragraphs, two examples are introduced to explain Definition 3.1.

Example 3.2. Let $\mathcal{M}=\{a, b, c\}, R=\left\{r_{1}, r_{2}, r_{3}\right\}$ and $=\{x, y, z\}, \mathcal{K}=\left\{k_{1}, k_{2}\right\}$. Let $\tilde{u}: \mathcal{S} \mathcal{S}(\mathcal{M}, R) \rightarrow$ $\mathcal{S} \mathcal{S}(\mathcal{M}, R)$ and $\tilde{v}: \mathcal{S} \mathcal{S}(\mathcal{N}, \mathcal{K}) \rightarrow \mathcal{S} \mathcal{S}(\mathcal{N}, \mathcal{K})$ be soft-co's defined as follows:

$$
\begin{gathered}
\tilde{u}\left(\mathcal{F}_{R}\right)=\left\{\begin{array}{cl}
\widetilde{\Phi}_{R} & \text { if } \mathcal{F}_{R} \subseteq \widetilde{\Phi}_{R}, \\
\left.\left(r_{1},\{a\}\right),\left(r_{2},\{b\}\right)\right\} & \text { if } \mathcal{F}_{R} \sqsubseteq\left\{\left(r_{1},\{a\},\left(r_{2},\{b\}\right)\right\},\right. \\
\widetilde{\mathcal{M}} & \text { otherwise. }
\end{array}\right. \\
\tilde{v}\left(G_{\mathcal{K}}\right)=\left\{\begin{array}{cl}
\widetilde{\Phi}_{\mathcal{K}} & \text { if } G_{\mathcal{K}} \sqsubseteq \widetilde{\Phi}_{\mathcal{K}}, \\
\left\{\left(k_{1},\{x, y\}\right),\left(k_{2},\{x, y\}\right)\right\} & \text { if } G_{\mathcal{K}} \sqsubseteq\left\{\left(k_{1},\{x, y\}\right),\left(k_{2},\{x, y\}\right)\right\}, \\
\widetilde{\mathcal{N}} & \text { otherwise. }
\end{array}\right.
\end{gathered}
$$

Clearly, $(\mathcal{M}, \tilde{u}, R)$ and $(\mathcal{N}, \tilde{v}, \mathcal{K})$ are soft-cs's. Then, the soft mapping $\psi_{\ell}:(\mathcal{M}, \tilde{u}, R) \rightarrow(\mathcal{N}, \tilde{v}, \mathcal{K})$ defined by $\psi(a)=x, \psi(b)=y, \psi(c)=z$ and $\ell\left(r_{1}\right)=k_{1}, \ell\left(r_{2}\right)=\ell\left(r_{3}\right)=k_{2}$ is a soft continuous mapping.

Example 3.3. Let $\mathcal{M}=\{a, b, c\}, R=\left\{r_{1}, r_{2}\right\}$ and $=\{x, y, z\}, \mathcal{K}=\left\{k_{1}, k_{2}\right\}$. Let $\tilde{u}: \mathcal{S} \mathcal{S}(\mathcal{M}, R) \rightarrow$ $\mathcal{S} \mathcal{S}(\mathcal{M}, R)$ and $\tilde{v}: \mathcal{S} \mathcal{S}(\mathcal{N}, \mathcal{K}) \rightarrow \mathcal{S} \mathcal{S}(\mathcal{N}, \mathcal{K})$ be soft-co's defined as follows:

$$
\begin{gathered}
\tilde{u}\left(\mathcal{F}_{R}\right)= \begin{cases}\widetilde{\Phi}_{R} & \text { if } \mathcal{F}_{R}=\widetilde{\Phi}_{R} \\
\left\{\left(r_{1},\{b\}\right)\right\} & \text { if } \mathcal{F}_{R}=\left\{\left(r_{1},\{b\}\right)\right\} \\
\widetilde{\mathcal{M}} & \text { otherwise. }\end{cases} \\
\tilde{v}\left(G_{\mathcal{K}}\right)=\left\{\begin{array}{cc}
\widetilde{\Phi}_{\mathcal{K}} & \text { if } G_{\mathcal{K}}=\widetilde{\Phi}_{\mathcal{K}} \\
\left\{\left(k_{1},\{x, y\}\right)\right\} & \text { if } G_{\mathcal{K}}=\left\{\left(k_{1},\{x\}\right)\right\}, \\
\left\{\left(k_{1},\{y\}\right)\right\} & \text { if } G_{\mathcal{K}}=\left\{\left(k_{1},\{y\}\right)\right\}, \\
\widetilde{\mathcal{N}} & \text { otherwise. }
\end{array}\right.
\end{gathered}
$$

Clearly, $(\mathcal{M}, \tilde{u}, R)$ and $(\mathcal{N}, \tilde{v}, \mathcal{K})$ are soft-cs's. Then, the soft mapping $\psi_{\ell}:(\mathcal{M}, \tilde{u}, R) \rightarrow(\mathcal{N}, \tilde{v}, \mathcal{K})$ defined by $\psi(a)=x, \psi(b)=z, \psi(c)=y$ and $\ell\left(r_{1}\right)=k_{2}, \ell\left(r_{2}\right)=k_{1}$ is not soft continuous mapping. Since there exists a soft set $\mathcal{F}_{R}=\left\{\left(r_{2},\{c\}\right)\right\} \in \mathcal{S} \mathcal{S}(\mathcal{M}, R)$, such that 
$\psi_{\ell}\left(\tilde{u}\left(\mathcal{F}_{R}\right)\right)=\tilde{\mathcal{N}} \nsubseteq\left\{\left(k_{1},\{y\}\right)\right\}=\tilde{v}\left(\psi_{\ell}\left(\mathcal{F}_{R}\right)\right)$.

Proposition 3.4. Let $(\mathcal{M}, \tilde{u}, R)$ and $(\mathcal{N}, \tilde{v}, \mathcal{K})$ be soft-cs's. If $\psi_{\ell}:(\mathcal{M}, \tilde{u}, R) \rightarrow(\mathcal{N}, \tilde{v}, \mathcal{K})$ is soft continuous mapping, then $\tilde{u}\left(\psi_{\ell}^{-1}\left(G_{\mathcal{K}}\right)\right) \subseteq \psi_{\ell}^{-1}\left(\tilde{v}\left(G_{\mathcal{K}}\right)\right)$ for every soft set $G_{\mathcal{K}} \in \mathcal{S} \mathcal{S}(\mathcal{N}, \mathcal{K})$.

Proof. Let $G_{\mathcal{K}} \in \mathcal{S} \mathcal{S}(\mathcal{N}, \mathcal{K})$. Then, $\psi_{\ell}^{-1}\left(G_{\mathcal{K}}\right) \in \mathcal{S} \mathcal{S}(\mathcal{M}, R)$. From the hypothesis, we obtain $\psi_{\ell}\left(\tilde{u}\left(\psi_{\ell}^{-1}\left(G_{\mathcal{K}}\right)\right) \sqsubseteq \tilde{v}\left(\psi_{\ell}\left(\psi_{\ell}^{-1}\left(G_{\mathcal{K}}\right)\right)\right) \sqsubseteq \tilde{v}\left(G_{\mathcal{K}}\right)\right.$. Consequently, by taking the inverse image we get, $\psi_{\ell}^{-1}\left(\psi_{\ell}\left(\tilde{u}\left(\psi_{\ell}^{-1}\left(G_{\mathcal{K}}\right)\right)\right) \sqsubseteq \psi_{\ell}^{-1}\left(\tilde{v}\left(G_{\mathcal{K}}\right)\right)\right.$. Hence, $\tilde{u}\left(\psi_{\ell}^{-1}\left(G_{\mathcal{K}}\right)\right) \sqsubseteq \psi_{\ell}^{-1}\left(\tilde{v}\left(G_{\mathcal{K}}\right)\right)$.

Proposition 3.5. If $\psi_{\ell}:(\mathcal{M}, \tilde{u}, R) \rightarrow(\mathcal{N}, \tilde{v}, \mathcal{K})$ is a soft continuous mapping, then $\psi_{\ell}^{-1}\left(G_{\mathcal{K}}\right)$ is a closed soft set of $(\mathcal{M}, \tilde{u}, R)$ for every closed soft set $G_{\mathcal{K}}$ of $(\mathcal{N}, \tilde{v}, \mathcal{K})$.

Proof: Let $G_{\mathcal{K}}$ be a closed soft set of $(\mathcal{N}, \tilde{v}, \mathcal{K})$. From $\psi_{\ell}$ is a soft continuous mapping and by Proposition 3.4, we have $\tilde{u}\left(\psi_{\ell}^{-1}\left(G_{\mathcal{K}}\right)\right) \sqsubseteq \psi_{\ell}^{-1}\left(\tilde{v}\left(G_{\mathcal{K}}\right)\right)$. Since $G_{\mathcal{K}}$ is a closed soft set, $\tilde{u}\left(\psi_{\ell}^{-1}\left(G_{\mathcal{K}}\right)\right) \sqsubseteq \psi_{\ell}^{-1}\left(G_{\mathcal{K}}\right)$ and from $(C 2)$ of Definition 2.12, we obtain $\tilde{u}\left(\psi_{\ell}^{-1}\left(G_{\mathcal{K}}\right)\right)=\psi_{\ell}^{-1}\left(G_{\mathcal{K}}\right)$. Therefore, $\psi_{\ell}^{-1}\left(G_{\mathcal{K}}\right)$ is a closed soft set of $(\mathcal{M}, \tilde{u}, R)$.

Corollary 3.6. If $\psi_{\ell}:(\mathcal{M}, \tilde{u}, R) \rightarrow(\mathcal{N}, \tilde{v}, \mathcal{K})$ is a soft continuous mapping, then $\psi_{\ell}^{-1}\left(G_{\mathcal{K}}\right)$ is an open soft set of $(\mathcal{M}, \tilde{u}, R)$ for every open soft set $G_{\mathcal{K}}$ of $(\mathcal{N}, \tilde{v}, \mathcal{K})$.

Remark 3.7. The converse of Proposition 3.5 and Corollary 3.6 may not be true.

Example 3.8. Let $\mathcal{M}=\{a, b, c\}, R=\left\{r_{1}, r_{2}\right\}$ and $=\{x, y, z\}, \mathcal{K}=\left\{k_{1}, k_{2}\right\}$. Let $\tilde{u}: \mathcal{S} \mathcal{S}(\mathcal{M}, R) \rightarrow$ $\mathcal{S} \mathcal{S}(\mathcal{M}, R)$ and $\tilde{v}: \mathcal{S} \mathcal{S}(\mathcal{N}, \mathcal{K}) \rightarrow \mathcal{S} \mathcal{S}(\mathcal{N}, \mathcal{K})$ be soft-co's defined as follows:

$$
\begin{aligned}
& \tilde{u}\left(\mathcal{F}_{R}\right)= \begin{cases}\widetilde{\Phi}_{R} & \text { if } \mathcal{F}_{R}=\widetilde{\Phi}_{R}, \\
\left\{\left(r_{1},\{c\}\right)\right\} & \text { if } \mathcal{F}_{R}=\left\{\left(r_{1},\{c\}\right\},\right. \\
\left\{\left(r_{2},\{a, b\}\right)\right\} & \text { if } \mathcal{F}_{R}=\left\{\left(r_{2},\{a\}\right)\right\}, \\
\widetilde{\mathcal{M}} & \text { otherwise. }\end{cases} \\
& \tilde{v}\left(G_{\mathcal{K}}\right)= \begin{cases}\widetilde{\Phi}_{\mathcal{K}} & \text { if } G_{\mathcal{K}}=\widetilde{\Phi}_{\mathcal{K}}, \\
\left\{\left(k_{1},\{y\}\right)\right\} & \text { if } G_{\mathcal{K}}=\left\{\left(k_{1},\{y\}\right)\right\}, \\
\left\{\left(k_{2},\{x, y\}\right)\right\} & \text { if } G_{\mathcal{K}}=\left\{\left(k_{2},\{x\}\right)\right\}, \\
\widetilde{\mathcal{N}} & \text { otherwise. }\end{cases}
\end{aligned}
$$

Clearly, $(\mathcal{M}, \tilde{u}, R)$ and $(\mathcal{N}, \tilde{v}, \mathcal{K})$ are soft-cs's. Let $\psi_{\ell}:(\mathcal{M}, \tilde{u}, R) \rightarrow(\mathcal{N}, \tilde{v}, \mathcal{K})$ be a soft mapping defined by $\psi(a)=x, \psi(b)=z, \psi(c)=y$ and $\ell\left(r_{1}\right)=k_{1}, \ell\left(r_{2}\right)=k_{2}$. Then, it is clear that, for any closed soft set $G_{\mathcal{K}}$ of $(\mathcal{N}, \tilde{v}, \mathcal{K}), \psi_{\ell}^{-1}\left(G_{K}\right)$ is a closed soft set in $(\mathcal{M}, \tilde{u}, R)$. However, there exists a soft $\quad$ set $\mathcal{F}_{R}=\left\{\left(r_{2},\{a\}\right)\right\} \in \mathcal{S} \mathcal{S}(\mathcal{M}, R)$ such that $\psi_{\ell}\left(\tilde{u}\left(\mathcal{F}_{R}\right)\right)=\left\{\left(k_{2},\{x, z\}\right)\right\} \nsubseteq\left\{\left(k_{2},\{x, y\}\right)\right\}=$ $\tilde{v}\left(\psi_{\ell}\left(\mathcal{F}_{R}\right)\right)$. Hence, $\psi_{\ell}$ is not soft continuous.

Proposition 3.9. Let $(\mathcal{M}, \tilde{u}, R),(\mathcal{N}, \tilde{v}, \mathcal{K})$, and $(\mathcal{Z}, \widetilde{w}, \mathcal{Q})$ be soft-cs's. If $\psi_{\ell}:(\mathcal{M}, \tilde{u}, R) \rightarrow(\mathcal{N}, \tilde{v}, \mathcal{K})$ and $\varphi_{q}:(\mathcal{N}, \tilde{v}, \mathcal{K}) \rightarrow(\mathcal{Z}, \widetilde{w}, \mathcal{Q})$ are soft continuous mappings, then $\varphi_{q} o \psi_{\ell}:(\mathcal{M}, \tilde{u}, R) \rightarrow(\mathcal{Z}, \widetilde{w}, \mathcal{Q})$ is a soft continuous mapping.

Proof: Let $\mathcal{F}_{R} \in \mathcal{S} \mathcal{S}(\mathcal{M}, R)$. By the definition of composition $\varphi_{q} o \psi_{\ell}\left(\tilde{u}\left(\mathcal{F}_{R}\right)\right)=\varphi_{q}\left(\psi_{\ell}\left(\tilde{u}\left(\mathcal{F}_{R}\right)\right)\right.$ and since $\psi_{\ell}$ is soft continuous, then it follows that $\varphi_{q}\left(\psi_{\ell}\left(\left(\tilde{u}\left(\mathcal{F}_{R}\right)\right) \subseteq \varphi_{q}\left(\tilde{v}\left(\psi_{\ell}\left(\mathcal{F}_{R}\right)\right)\right.\right.\right.$. As $\varphi_{q}$ is soft continuous, we get $\varphi_{q}\left(\tilde{v}\left(\psi_{\ell}\left(\mathcal{F}_{R}\right)\right) \sqsubseteq \widetilde{w}\left(\varphi_{q}\left(\psi_{\ell}\left(\mathcal{F}_{R}\right)\right)\right.\right.$. Consequently, $\varphi_{q} o \psi_{\ell}\left(\tilde{u}\left(\mathcal{F}_{R}\right)\right) \sqsubseteq$ $\widetilde{w}\left(\varphi_{q} o \psi_{\ell}\left(\mathcal{F}_{R}\right)\right)$.

Hence, $\varphi_{q} o \psi_{\ell}$ is a soft continuous mapping.

Proposition 3.10. Let $(\mathcal{M}, \tilde{u}, R)$ and $(\mathcal{N}, \tilde{v}, \mathcal{K})$ be soft-cs's and let $\left(\mathcal{Y}, \tilde{u}_{y}, R\right)$ be a closed soft-c.subsp of $(\mathcal{M}, \tilde{u}, R)$. If $\psi_{\ell}:(\mathcal{M}, \tilde{u}, R) \rightarrow(\mathcal{N}, \tilde{v}, \mathcal{K})$ is soft continuous mapping, then the restriction mapping $\left.\psi_{\ell}\right|_{y}:\left(\mathcal{Y}, \tilde{u}_{y}, R\right) \rightarrow(\mathcal{N}, \tilde{v}, \mathcal{K})$ is soft continuous mapping.

Proof: We must prove that, for all $\mathcal{F}_{R} \in \mathcal{S} \mathcal{S}\left(\mathcal{Y}, \tilde{u}_{y}, R\right),\left.\psi_{\ell}\right|_{y}\left(\tilde{u}_{y}\left(\mathcal{F}_{R}\right)\right) \sqsubseteq \tilde{v}\left(\left.\psi_{\ell}\right|_{y}\left(\mathcal{F}_{R}\right)\right)$. Now,

$$
\begin{aligned}
\left.\psi_{\ell}\right|_{y}\left(\tilde{u}_{y}\left(\mathcal{F}_{R}\right)\right) & =\left.\psi_{\ell}\right|_{y}\left(\tilde{\mathcal{Y}} \sqcap \tilde{u}\left(\mathcal{F}_{R}\right)\right) & & \text { (by definition of } \left.\tilde{u}_{y}\right) \\
& =\left.\psi_{\ell}\right|_{y}\left(\tilde{u}\left(\mathcal{F}_{R}\right)\right) & & \left(\text { by }(C 2) \mathcal{F}_{R} \sqsubseteq \tilde{\mathcal{y}} \Rightarrow \tilde{u}\left(\mathcal{F}_{R}\right) \sqsubseteq \tilde{u}(\tilde{\mathcal{Y}})=\tilde{\mathcal{Y}}\right) \\
& =\psi_{\ell}\left(\tilde{u}\left(\mathcal{F}_{R}\right)\right) & & \left(\text { by } \tilde{u}\left(\mathcal{F}_{R}\right) \sqsubseteq \tilde{\mathcal{Y}}\right)
\end{aligned}
$$




$$
=\tilde{v}\left(\psi_{\ell}\left(G_{R}\right)\right) \quad \text { (by } \psi_{\ell} \text { is soft continuous). }
$$

Hence, $\left.\psi_{\ell}\right|_{y}$ is a soft continuous mapping.

Definition 3.11. Let $(\mathcal{M}, \tilde{u}, R)$ and $(\mathcal{N}, \tilde{v}, \mathcal{K})$ be soft-cs's. A soft mapping $\psi_{\ell}:(\mathcal{M}, \tilde{u}, R) \rightarrow(\mathcal{N}, \tilde{v}, \mathcal{K})$ is said to be soft closed (resp. open), if $\psi_{\ell}\left(\mathcal{F}_{R}\right)$ is closed (resp. open) soft set of $(\mathcal{N}, \tilde{v}, \mathcal{K})$ whenever $\mathcal{F}_{R}$ is closed (resp. open) soft set of $(\mathcal{M}, \tilde{u}, R)$.

Example 3.12 Let $\mathcal{M}=\{a, b, c\}, R=\left\{r_{1}, r_{2}\right\}$ and $\mathcal{N}=\{x, y, z\}, \mathcal{K}=\left\{k_{1}, k_{2}\right\}$. Let $\tilde{u}: \mathcal{S} \mathcal{S}(\mathcal{M}, R) \rightarrow$ $\mathcal{S} \mathcal{S}(\mathcal{M}, R)$ and $\tilde{v}: \mathcal{S} \mathcal{S}(\mathcal{N}, \mathcal{K}) \rightarrow \mathcal{S} \mathcal{S}(\mathcal{N}, \mathcal{K})$ be soft-co's defined as follows:

$$
\begin{gathered}
\tilde{u}\left(\mathcal{F}_{R}\right)=\left\{\begin{array}{cl}
\widetilde{\Phi}_{R} & \text { if } \mathcal{F}_{R}=\widetilde{\Phi}_{R}, \\
\left\{\left(r_{1},\{b, c\}\right),\left(r_{2},\{b, c\}\right)\right\} & \text { if } \mathcal{F}_{R} \sqsubseteq\left\{\left(r_{1},\{b, c\}\right),\left(r_{2},\{b, c\}\right)\right\}, \\
\widetilde{\mathcal{M}} & \text { otherwise. }
\end{array}\right. \\
\tilde{v}\left(G_{\mathcal{K}}\right)=\left\{\begin{array}{cl}
\widetilde{\Phi}_{\mathcal{K}} & \text { if } G_{\mathcal{K}}=\widetilde{\Phi}_{\mathcal{K}}, \\
\left\{\left(k_{1},\{y, z\}\right),\left(k_{2},\{y, z\}\right)\right\} & \text { if } G_{\mathcal{K}} \sqsubseteq\left\{\left(k_{1},\{y, z\}\right),\left(k_{2},\{y, z\}\right)\right\}, \\
\widetilde{\mathcal{N}} & \text { otherwise. }
\end{array}\right.
\end{gathered}
$$

Clearly, $(\mathcal{M}, \tilde{u}, R)$ and $(\mathcal{N}, \tilde{v}, \mathcal{K})$ are soft-cs's. Then, the soft mapping $\psi_{\ell}:(\mathcal{M}, \tilde{u}, R) \rightarrow(\mathcal{N}, \tilde{v}, \mathcal{K})$ defined by $\psi(a)=\psi(b)=\psi(c)=x$ and $\ell\left(r_{1}\right)=k_{1}, \ell\left(r_{2}\right)=k_{2}$ is a soft open mapping. But it is not soft closed mapping since there exists a closed soft set $\mathcal{F}_{R}=\left\{\left(r_{1},\{b, c\}\right),\left(r_{2},\{b, c\}\right)\right\}$, but $\psi_{\ell}\left(\mathcal{F}_{R}\right)=\left\{\left(k_{1},\{x\}\right),\left(k_{2},\{x\}\right)\right\}$ is not closed soft set in $(\mathcal{N}, \tilde{v}, \mathcal{K})$.

Proposition 3.13. Let $(\mathcal{M}, \tilde{u}, R)$ and $(\mathcal{N}, \tilde{v}, \mathcal{K})$ be soft-cs's. Let $\psi_{\ell}$ be a soft mapping from $(\mathcal{M}, \tilde{u}, R)$ onto $(\mathcal{N}, \tilde{v}, \mathcal{K})$. Then $\psi_{\ell}$ is soft closed if and only if, for each soft set $\mathcal{F}_{\mathcal{K}} \in \mathcal{S} \mathcal{S}(\mathcal{N}, \mathcal{K})$ and each open soft set $G_{R}$ of $(\mathcal{M}, \tilde{u}, R)$ containing $\psi_{\ell}^{-1}\left(\mathcal{F}_{\mathcal{K}}\right)$, there is an open soft set $H_{\mathcal{K}}$ of $(\mathcal{N}, \tilde{v}, \mathcal{K})$ such that $\mathcal{F}_{\mathcal{K}} \sqsubseteq H_{\mathcal{K}}$ and $\psi_{\ell}^{-1}\left(H_{\mathcal{K}}\right) \sqsubseteq G_{R}$.

Proof: Let $\psi_{\ell}$ be a soft closed mapping, $\mathcal{F}_{\mathcal{K}} \in \mathcal{S} \mathcal{S}(\mathcal{N}, \mathcal{K})$, and let $G_{R}$ be an open soft set of $(\mathcal{M}, \tilde{u}, R)$ such that $\psi_{\ell}^{-1}\left(\mathcal{F}_{\mathcal{K}}\right) \sqsubseteq G_{R}$. Then, $\psi_{\ell}\left(\widetilde{\mathcal{M}}-G_{R}\right)$ is a closed soft set of $(\mathcal{N}, \tilde{v}, \mathcal{K})$. Let $H_{\mathcal{K}}=\widetilde{\mathcal{N}}-$ $\psi_{\ell}\left(\tilde{\mathcal{M}}-G_{R}\right)$. Then, $H_{\mathcal{K}}$ is an open soft set of $(\mathcal{N}, \tilde{v}, \mathcal{K})$ and $\psi_{\ell}^{-1}\left(H_{\mathcal{K}}\right)=\psi_{\ell}^{-1}\left(\widetilde{\mathcal{N}}-\psi_{\ell}(\tilde{\mathcal{M}}-\right.$ $\left.\left.G_{R}\right)\right)=\tilde{\mathcal{M}}-\psi_{\ell}^{-1}\left(\psi_{\ell}\left(\tilde{\mathcal{M}}-G_{R}\right)\right) \sqsubseteq \tilde{\mathcal{M}}-\left(\tilde{\mathcal{M}}-G_{R}\right)=G_{R}$. Thus, $H_{\mathcal{K}}$ is an open soft set of $(\mathcal{N}, \tilde{v}, \mathcal{K})$ containing $\mathcal{F}_{\mathcal{K}}$ such that $\psi_{\ell}^{-1}\left(\mathcal{F}_{\mathcal{K}}\right) \subseteq G_{R}$

Conversely, let $\mathcal{F}_{R}$ be a closed soft set of $(\mathcal{M}, \tilde{u}, R)$. Then, $\psi_{\ell}^{-1}\left(\tilde{\mathcal{N}}-\psi_{\ell}\left(\mathcal{F}_{R}\right)\right) \subseteq \tilde{\mathcal{M}}-\mathcal{F}_{R}$ and $\tilde{\mathcal{M}}-\mathcal{F}_{R}$ is an open soft of $(\mathcal{M}, \tilde{u}, R)$. From the hypothesis, there is an open soft set $H_{\mathcal{K}}$ of $(\mathcal{N}, \tilde{v}, \mathcal{K})$ such that $\tilde{\mathcal{N}}-\psi_{\ell}\left(\mathcal{F}_{R}\right) \sqsubseteq H_{\mathcal{K}}$ and $\psi_{\ell}^{-1}\left(H_{\mathcal{K}}\right) \sqsubseteq \tilde{\mathcal{M}}-\mathcal{F}_{R}$. Therefore, $\mathcal{F}_{R} \sqsubseteq \tilde{\mathcal{M}}-\psi_{\ell}^{-1}\left(H_{\mathcal{K}}\right)$. Therefore, $\tilde{\mathcal{N}}-H_{\mathcal{K}} \sqsubseteq \psi_{\ell}\left(\mathcal{F}_{R}\right) \sqsubseteq \psi_{\ell}\left(\tilde{\mathcal{M}}-\psi_{\ell}^{-1}\left(H_{\mathcal{K}}\right)\right) \sqsubseteq \widetilde{\mathcal{N}}-H_{\mathcal{K}}$, which yields $\psi_{\ell}\left(\mathcal{F}_{R}\right)=\widetilde{\mathcal{N}}-H_{\mathcal{K}}$. Therefore, $\psi_{\ell}\left(\mathcal{F}_{R}\right)$ is a closed soft set of $(\mathcal{N}, \tilde{v}, \mathcal{K})$. Hence, $\psi_{\ell}$ is a soft closed mapping.

Proposition 3.14. Let $(\mathcal{M}, \tilde{u}, R),(\mathcal{N}, \tilde{v}, \mathcal{K})$ and $(\mathcal{Z}, \widetilde{w}, \mathcal{Q})$ be soft-cs's and let $\psi_{\ell}:(\mathcal{M}, \tilde{u}, R) \rightarrow$ $(\mathcal{N}, \tilde{v}, \mathcal{K})$ and $\varphi_{q}:(\mathcal{N}, \tilde{v}, \mathcal{K}) \rightarrow(\mathcal{Z}, \widetilde{w}, \mathcal{Q})$ be soft mappings. Then

i- $\quad$ if $\psi_{\ell}$ and $\varphi_{q}$ are soft closed, then so is $\varphi_{q} o \psi_{\ell}$.

ii- if $\varphi_{q} o \psi_{\ell}$ is soft closed and $\psi_{\ell}$ is soft continuous and surjection, then $\varphi_{q}$ is a soft closed mapping.

iii- if $\varphi_{q} o \psi_{\ell}$ is soft closed and $\varphi_{q}$ is soft continuos and injection, then $\psi_{\ell}$ is a soft closed mapping.

\section{Proof}

i- $\quad$ Let $\mathcal{F}_{R}$ be a closed soft set of $(\mathcal{M}, \tilde{u}, R)$. By $\psi_{\ell}$ is soft closed mapping, we get $\psi_{\ell}\left(\mathcal{F}_{R}\right)$ closed soft set in $(\mathcal{N}, \tilde{v}, \mathcal{K})$. Since $\varphi_{q}$ is closed soft, then $\varphi_{q}\left(\psi_{\ell}\left(\mathcal{F}_{R}\right)\right)$ is closed soft set in $(\mathcal{Z}, \widetilde{w}, \mathcal{Q})$. Hence, $\varphi_{q} o \psi_{\ell}$ is soft closed mapping.

ii- Let $G_{\mathcal{K}}$ be a soft closed set of $(\mathcal{N}, \tilde{v}, \mathcal{K})$. Since $\psi_{\ell}$ is a soft continuous, then $\psi_{\ell}^{-1}\left(G_{\mathcal{K}}\right)$ is soft closed in $(\mathcal{M}, \tilde{u}, R)$. Since $\varphi_{q} o \psi_{\ell}$ is soft closed, then $\varphi_{q} o \psi_{\ell}\left(\psi_{\ell}^{-1}\left(G_{\mathcal{K}}\right)\right)=\varphi_{q}\left(\psi_{\ell}\left(\psi_{\ell}^{-1}\left(G_{\mathcal{K}}\right)\right)\right)$ is a soft closed set in $(\mathcal{Z}, \widetilde{w}, \mathcal{Q})$. But $\psi_{\ell}$ is surjection, then $\varphi_{q} o \psi_{\ell}\left(\psi_{\ell}^{-1}\left(G_{\mathcal{K}}\right)\right)=\varphi_{q}\left(\psi_{\ell}\left(\psi_{\ell}^{-1}\left(G_{\mathcal{K}}\right)\right)\right)=$ $\varphi_{q}\left(G_{\mathcal{K}}\right)$. Consequently, $\varphi_{q}\left(G_{\mathcal{K}}\right)$ is a soft closed set in $(\mathcal{Z}, \widetilde{w}, \mathcal{Q})$. Hence, $\varphi_{q}$ is soft closed mapping. 
iii- $\quad$ Let $\mathcal{F}_{R}$ be closed in $(\mathcal{M}, \tilde{u}, R)$ to prove that $\psi_{\ell}\left(\mathcal{F}_{R}\right)$ closed in $(\mathcal{N}, \tilde{v}, \mathcal{K})$. Since $\varphi_{q} o \psi_{\ell}$ is soft closed, then $\left(\varphi_{q} \circ \psi_{\ell}\right)\left(\mathcal{F}_{R}\right)$ is closed in $(\mathcal{Z}, \widetilde{w}, \mathcal{Q})$ and since $\varphi_{q}$ is soft continuous, then $\varphi_{q}^{-1}\left(\left(\varphi_{q} o \psi_{\ell}\right)\left(\mathcal{F}_{R}\right)\right)$ is closed of $(\mathcal{N}, \tilde{v}, \mathcal{K})$. This implies that $\varphi_{q}^{-1}\left(\varphi_{q}\left(\psi_{\ell}\left(\mathcal{F}_{R}\right)\right)\right.$ is a closed soft set of $(\mathcal{N}, \tilde{v}, \mathcal{K})$. Since $\varphi_{q}$ is one to one, then $\psi_{\ell}\left(\mathcal{F}_{R}\right)$ is closed soft in $(\mathcal{N}, \tilde{v}, \mathcal{K})$.

\section{Soft continuous mappings between product soft closure spaces}

In this section, we study some properties of soft continuous mappings in the product soft closure space. First, we show that the soft projection map is soft closed and continuous.

Theorem 4.1. Let $\left\{\left(\mathcal{M}_{\alpha}, \tilde{u}_{\alpha}, R_{\alpha}\right): \alpha \in \mathcal{J}\right\}$ be a family of soft-cs's and let $v \in \mathcal{J}$. Then, the soft projection mapping $\left(p_{\mathcal{M}_{v}}, q_{R_{v}}\right):\left(\prod_{\alpha \in \mathcal{J}} \mathcal{M}_{\alpha}, \otimes \tilde{u}, \prod_{\alpha \in \mathcal{J}} R_{\alpha}\right) \rightarrow\left(\mathcal{M}_{v}, \tilde{u}_{v}, R_{v}\right)$ is a soft closed and soft continuous mapping.

Proof. First, we prove that for all $v \in \mathcal{J}$, the soft projection mapping $\left(p_{\mathcal{M}_{v}}, q_{R_{v}}\right)$ is soft closed. Let

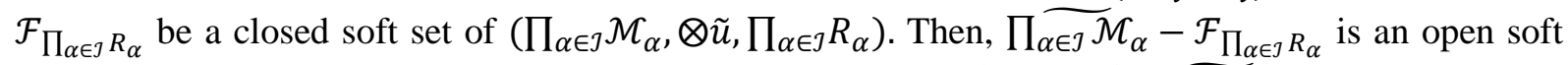
set of $\left(\prod_{\alpha \in \mathcal{J}} \mathcal{M}_{\alpha}, \otimes \tilde{u}, \prod_{\alpha \in \mathcal{J}} R_{\alpha}\right)$. From Theorem 2.15, we have $\left(p_{\mathcal{M}_{v}}, q_{R_{v}}\right)\left(\prod_{\alpha \in \mathcal{J}} \mathcal{M}_{\alpha}-\mathcal{F}_{\prod_{\alpha \in \mathcal{J}} R_{\alpha}}\right)$ is an open soft set of $\left(\mathcal{M}_{v}, \tilde{u}_{v}, R_{v}\right)$. But, $\left(p_{\mathcal{M}_{v}}, q_{R_{v}}\right)\left(\prod_{\alpha \in \mathcal{J}} \mathcal{M}_{\alpha}-\mathcal{F}_{\prod_{\alpha \in \mathcal{J}} R_{\alpha}}\right)=\widetilde{\mathcal{M}}_{v}-\left(p_{\mathcal{M}_{v}}, q_{R_{v}}\right)\left(\mathcal{F}_{\prod_{\alpha \in \mathcal{J}} R_{\alpha}}\right)$, which is an open soft set in $\left(\mathcal{M}_{v}, \tilde{u}_{v}, R_{v}\right)$. Hence, $\left(p_{\mathcal{M}_{v}}, q_{R_{v}}\right)\left(\mathcal{F}_{\prod_{\alpha \in J} R_{\alpha}}\right)$ is a closed soft set in $\left(\mathcal{M}_{v}, \tilde{u}_{v}, R_{v}\right)$. Thus, the soft projection map $\left(p_{\mathcal{M}_{v}}, q_{R_{v}}\right)$ is a soft closed mapping.

Now, we shall show that $\left(p_{\mathcal{M}_{v}}, q_{R_{v}}\right)$ is soft continuous mapping for all $v \in \mathcal{J}$. Let $G_{\prod_{\alpha \in \mathcal{J}} R_{\alpha}}$ 드 $\widetilde{\prod_{\alpha \in \mathcal{J}} \mathcal{M}_{\alpha}}$ to prove that $\left(p_{\mathcal{M}_{v}}, q_{R_{v}}\right)\left(\otimes \tilde{u}\left(G_{\prod_{\alpha \in \mathcal{J}} R_{\alpha}}\right)\right) \sqsubseteq \tilde{u}_{v}\left(\left(p_{\mathcal{M}_{v}}, q_{R_{v}}\right)\left(G_{\prod_{\alpha \in \mathcal{J}} R_{\alpha}}\right)\right.$. From the definition of $\otimes \tilde{u}$, we have

$$
\begin{gathered}
\left(p_{\mathcal{M}_{v}}, q_{R_{v}}\right)\left(\otimes \tilde{u}\left(G_{\prod_{\alpha \in \mathcal{J}} R_{\alpha}}\right)=\left(p_{\mathcal{M}_{v}}, q_{R_{v}}\right)\left(\prod_{\alpha \in \mathcal{J}} \tilde{u}_{\alpha}\left(\left(p_{\mathcal{M}_{\alpha}}, q_{R_{\alpha}}\right)\left(G_{\prod_{\alpha \in \mathcal{J}} R_{\alpha}}\right)\right)\right.\right. \\
=\tilde{u}_{v}\left(\left(p_{\mathcal{M}_{v}}, q_{R_{v}}\right)\left(G_{\prod_{\alpha \in \mathcal{J}} R_{\alpha}}\right) .\right.
\end{gathered}
$$

Therefore, $\left(p_{\mathcal{M}_{v}}, q_{R_{v}}\right)$ is soft continuous mapping.

Theorem 4.2. Let $\left\{\left(\mathcal{M}_{\alpha}, \tilde{u}_{\alpha}, R_{\alpha}\right): \alpha \in \mathcal{J}\right\}$ be a family of soft-cs's and let $v \in \mathcal{J}$. Then, $\mathcal{F}_{R_{v}}$ is a closed set of $\left(\mathcal{M}_{v}, \tilde{u}_{v}, R_{v}\right)$ if and only if $\mathcal{F}_{R_{v}} \times \widetilde{\prod_{\alpha \neq v} \mathcal{M}_{\alpha}}$ is a closed soft set in $\left(\prod_{\alpha \in \mathcal{J}} \mathcal{M}_{\alpha}, \otimes \tilde{u}, \prod_{\alpha \in \mathcal{J}} R_{\alpha}\right)$.

Proof. Let $v \in \mathcal{J}$ and let $\mathcal{F}_{R_{v}}$ be a soft closed set of $\left(\mathcal{M}_{v}, \tilde{u}_{v}, R_{v}\right)$. Then, from Theorem 4.1, $\left(p_{\mathcal{M}_{v}}, q_{R_{v}}\right)$ is soft continuous and, by Proposition 3.5, we have $\left(p_{\mathcal{M}_{v}}, q_{R_{v}}\right)^{-1}\left(\mathcal{F}_{R_{v}}\right)$ is a soft closed set of $\left(\prod_{\alpha \in \mathcal{J}} \mathcal{M}_{\alpha}, \otimes \tilde{u}, \prod_{\alpha \in \mathcal{J}} R_{\alpha}\right)$. But, $\left(p_{\mathcal{M}_{v}}, q_{R_{v}}\right)^{-1}\left(\mathcal{F}_{R_{v}}\right)=\mathcal{F}_{R_{v}} \times \underset{\substack{\alpha \neq v \\ \alpha \in \mathcal{J}}}{\underset{\mathcal{M}}{\alpha}} \mathcal{L}_{\alpha}$. This implies that $\mathcal{F}_{R_{v}} \times$ $\underset{\substack{\alpha \neq v \\ \alpha \in \mathcal{J}}}{\widetilde{\mathcal{M}}} \mathrm{\alpha}_{\alpha}$ is a soft closed set of $\left(\prod_{\alpha \in \mathcal{J}} \mathcal{M} \mathcal{M}_{\alpha}, \otimes \tilde{u}, \prod_{\alpha \in \mathcal{J}} R_{\alpha}\right)$.

Conversely, $\mathcal{F}_{R_{v}} \times \underset{\substack{\alpha \neq v \\ \alpha \in \mathcal{J}}}{ } \mathcal{M}_{\alpha}$ is a soft closed set of $\left(\prod_{\alpha \in \mathcal{J}} \mathcal{M}_{\alpha}, \otimes \tilde{u}, \prod_{\alpha \in \mathcal{J}} R_{\alpha}\right)$. From Theorem 4.1, this implies that, for all $v \in \mathcal{J}$, the soft projection $\left(p_{\mathcal{M}_{v}}, q_{R_{v}}\right)$ is soft closed mapping. This implies that $\left(p_{\mathcal{M}_{v}}, q_{R_{v}}\right)\left(\mathcal{F}_{R_{v}} \times \underset{\substack{\alpha \neq v \\ \alpha \in \mathcal{J}}}{\widetilde{M}} \mathcal{M}_{\alpha}\right)=\mathcal{F}_{R_{v}}$ is a soft closed set of $\left(\mathcal{M}_{v}, \tilde{u}_{v}, R_{v}\right)$

Theorem 4.3. Let $\left\{\left(\mathcal{M}_{\alpha}, \tilde{u}_{\alpha}, R_{\alpha}\right): \alpha \in \mathcal{J}\right\}$ be a family of soft-cs's and let $v \in \mathcal{J}$. Then, $G_{R_{v}}$ is an open soft set of $\left(\mathcal{M}_{v}, \tilde{u}_{v}, R_{v}\right)$ if and only if $G_{R_{v}} \times \underset{\alpha \in \mathcal{J}}{\prod_{\alpha \neq \mathcal{N}} \mathcal{M}_{\alpha}}$ is an open soft set of $\left(\prod_{\alpha \in \mathcal{J}} \mathcal{M} \mathcal{M}_{\alpha}, \otimes \tilde{u}, \prod_{\alpha \in \mathcal{J}} R_{\alpha}\right)$.

Proof: Let $v \in \mathcal{J}$ and let $G_{R_{v}}$ be an open soft set of $\left(\mathcal{M}_{v}, \tilde{u}_{v}, R_{v}\right)$. From Theorem $4.1,\left(p_{\mathcal{M}_{v}}, q_{R_{v}}\right)$ is soft continuous mapping, then $\left(p_{\mathcal{M}_{v}}, q_{R_{v}}\right)^{-1}\left(G_{R_{v}}\right)$ is an open soft set of $\left(\prod_{\alpha \in \mathcal{J}} \mathcal{M}_{\alpha}, \otimes \tilde{u}, \prod_{\alpha \in \mathcal{J}} R_{\alpha}\right)$. But,

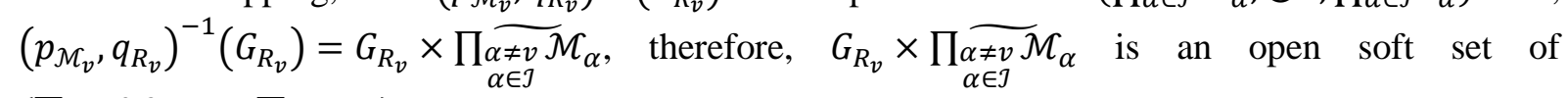
$\left(\prod_{\alpha \in \mathcal{J}} \mathcal{M}_{\alpha}, \otimes \tilde{u}, \prod_{\alpha \in \mathcal{J}} R_{\alpha}\right)$.

Conversely, let $G_{R_{v}} \times \prod_{\substack{\alpha \neq v \\ \alpha \in \mathcal{J}}} \mathcal{M}_{\alpha}$ be an open soft set of $\left(\prod_{\alpha \in \mathcal{J}} \mathcal{M}_{\alpha}, \otimes \tilde{u}, \prod_{\alpha \in \mathcal{J}} R_{\alpha}\right)$. Then, $\prod_{\substack{\alpha \neq v \\ \alpha \in \mathcal{J}}} \mathcal{M}_{\alpha}-$ $\left(G_{R_{v}} \times \prod_{\substack{\alpha \neq v \\ \alpha \in \mathcal{J}}} \mathcal{M}_{\alpha}\right)$ is a closed soft set of $\left(\prod_{\alpha \in \mathcal{J}} \mathcal{M}_{\alpha}, \otimes \tilde{u}, \prod_{\alpha \in \mathcal{J}} R_{\alpha}\right)$. But, $\underset{\substack{\alpha \neq v \\ \alpha \in \mathcal{J}}}{\widetilde{M}} \mathcal{C}_{\alpha}-\left(G_{R_{v}} \times\right.$ $\underset{\substack{\alpha \neq \mathcal{J} \\ \alpha \in \mathcal{M}}}{\alpha \in \mathcal{J}})=\left(\widetilde{\mathcal{M}_{v}}-G_{R_{v}}\right) \times \underset{\substack{\alpha \neq v \\ \alpha \in \mathcal{J}}}{\widetilde{\mathcal{M}}}{ }_{\alpha}$ is a closed soft set of $\left(\prod_{\alpha \in \mathcal{J}} \mathcal{M} \mathcal{M}_{\alpha}, \otimes \tilde{u}, \prod_{\alpha \in \mathcal{J}} R_{\alpha}\right)$. By Theorem 
4.2, $\left(\widetilde{\mathcal{M}_{v}}-G_{R_{v}}\right) \times \underset{\substack{\alpha \neq v \\ \alpha \in \mathcal{J}}}{\widetilde{\mathcal{M}}} \boldsymbol{T}_{\alpha}$ is a closed soft set of $\left(\prod_{\alpha \in \mathcal{J}} \mathcal{M}_{\alpha}, \otimes \tilde{u}, \prod_{\alpha \in \mathcal{J}} R_{\alpha}\right)$. Consequently, $G_{R_{v}}$ is an open soft set of $\left(\mathcal{M}_{v}, \tilde{u}_{v}, R_{v}\right)$.

Theorem 4.4. Let $(\mathcal{N}, \widetilde{\omega}, \mathcal{K})$ be a soft-cs, $\left\{\left(\mathcal{M}_{\alpha}, \tilde{u}_{\alpha}, R_{\alpha}\right): \alpha \in \mathcal{J}\right\}$ be a family of soft-cs's, and $\psi_{\ell}:(\mathcal{N}, \widetilde{\omega}, \mathcal{K}) \rightarrow\left(\prod_{\alpha \in \mathcal{J}} \mathcal{M}_{\alpha}, \otimes \tilde{u}, \prod_{\alpha \in \mathcal{J}} R_{\alpha}\right)$ be soft mapping. Then, $\psi_{\ell}$ is a soft closed mapping if and only if $\left(p_{\mathcal{M}_{\alpha}}, q_{R_{\alpha}}\right) o \psi_{\ell}$ is a soft closed mapping for each $\alpha \in \mathcal{J}$.

Proof. Let $\psi_{\ell}$ be a soft closed mapping. From Theorem 4.1, $\left(p_{\mathcal{M}_{\alpha}}, q_{R_{\alpha}}\right)$ is soft closed mapping and by Proposition 3.14 part (1), we get that $\left(p_{\mathcal{M}_{\alpha}}, q_{R_{\alpha}}\right) o \psi_{\ell}$ is a soft closed mapping for each $\alpha \in \mathcal{J}$.

Conversely, let $\left(p_{\mathcal{M}_{\alpha}}, q_{R_{\alpha}}\right) o \psi_{\ell}$ be a soft closed mapping for each $\alpha \in \mathcal{J}$. Suppose that $\psi_{\ell}$ is not a soft closed mapping. Then, there exists a closed soft set $\mathcal{F}_{\mathcal{K}}$ of $(\mathcal{N}, \widetilde{\omega}, \mathcal{K})$ such that $\psi_{\ell}\left(\mathcal{F}_{\mathcal{K}}\right)$ is not a closed soft set of $\left(\prod_{\alpha \in \mathcal{J}} \mathcal{M}_{\alpha}, \otimes \tilde{u}, \prod_{\alpha \in \mathcal{J}} R_{\alpha}\right)$, i.e., $\otimes \tilde{u}\left(\psi_{\ell}\left(\mathcal{F}_{\mathcal{K}}\right)\right) \neq \psi_{\ell}\left(\mathcal{F}_{\mathcal{K}}\right)$, which implies that $\otimes \tilde{u}\left(\psi_{\ell}\left(\mathcal{F}_{\mathcal{K}}\right)\right) \nsubseteq \psi_{\ell}\left(\mathcal{F}_{\mathcal{K}}\right)$. It follows that $\left.\prod_{\alpha \in \mathcal{J}} \tilde{u}_{\alpha}\left(p_{\mathcal{M}_{\alpha}}, q_{R_{\alpha}}\right)\left(\psi_{\ell}\left(\mathcal{F}_{\mathcal{K}}\right)\right)\right) \nsubseteq \psi_{\ell}\left(\mathcal{F}_{\mathcal{K}}\right)$. This implies that there exists $v \in \mathcal{J}$ such that $\tilde{u}_{v}\left(\left(p_{\mathcal{M}_{v}}, q_{R_{v}}\right)\right)\left(\psi_{\ell}\left(\mathcal{F}_{\mathcal{K}}\right)\right) \nsubseteq\left(p_{\mathcal{M}_{\alpha}}, q_{R_{\alpha}}\right)\left(\psi_{\ell}\left(\mathcal{F}_{\mathcal{K}}\right)\right)$. Since $\mathcal{F}_{\mathcal{K}}$ is a closed soft set of $(\mathcal{N}, \widetilde{\omega}, \mathcal{K})$ and $\left(p_{\mathcal{M}_{\alpha}}, q_{R_{\alpha}}\right) o \psi_{\ell}$ is a soft closed map for each $\alpha \in \mathcal{J}$, then $\left(\left(p_{\mathcal{M}_{v}}, q_{R_{v}}\right) o \psi_{\ell}\right)\left(\mathcal{F}_{\mathcal{K}}\right)$ is a closed soft set of $\left(\mathcal{M}_{v}, \tilde{u}_{v}, R_{v}\right)$. This implies that $\left(p_{\mathcal{M}_{v}}, q_{R_{v}}\right)\left(\psi_{\ell}\left(\mathcal{F}_{\mathcal{K}}\right)\right)$ is a closed soft set of $\left(\mathcal{M}_{v}, \tilde{u}_{v}, R_{v}\right)$. Consequently, $\tilde{u}_{v}\left(\left(p_{\mathcal{M}_{v}}, q_{R_{v}}\right)\right)\left(\psi_{\ell}\left(\mathcal{F}_{\mathcal{K}}\right)\right)=\left(p_{\mathcal{M}_{v}}, q_{R_{v}}\right)\left(\psi_{\ell}\left(\mathcal{F}_{\mathcal{K}}\right)\right)$ and this is a contradiction.

Theorem 4.5. Let $\left\{\left(\mathcal{M}_{\alpha}, \tilde{u}_{\alpha}, R_{\alpha}\right): \alpha \in \mathcal{J}\right\}$ and $\left\{\left(\mathcal{N}_{\alpha}, \widetilde{\omega}_{\alpha}, \mathcal{K}_{\alpha}\right): \alpha \in \mathcal{J}\right\}$ be families of soft-cs's. For each $\alpha \in \mathcal{J}$, let $\left(\psi_{\ell}\right)_{\alpha}:\left(\mathcal{M}_{\alpha}, \tilde{u}_{\alpha}, R_{\alpha}\right) \rightarrow\left(\mathcal{N}_{\alpha}, \widetilde{\omega}_{\alpha}, \mathcal{K}_{\alpha}\right)$ be a surjection and let $\psi_{\ell}:\left(\prod_{\alpha \in \mathcal{J}} \mathcal{M}_{\alpha}, \otimes \tilde{u}, \prod_{\alpha \in \mathcal{J}} R_{\alpha}\right) \rightarrow\left(\prod_{\alpha \in \mathcal{J}} \mathcal{N}_{\alpha}, \otimes \widetilde{\omega}, \prod_{\alpha \in \mathcal{J}} \mathcal{K}_{\alpha}\right), \quad$ defined $\quad$ by $\quad \psi_{\ell}\left(\left(x_{\alpha_{r_{\alpha}}}\right)_{\alpha \in \mathcal{J}}\right)=$ $\left(\left(\psi_{\ell}\right)_{\alpha}\left(x_{\alpha_{r_{\alpha}}}\right)\right)_{\alpha \in \mathcal{J}}$. Then, $\psi_{\ell}$ is soft closed if and only if $\left(\psi_{\ell}\right)_{\alpha}$ is soft closed mapping for each $\alpha \in \mathcal{J}$.

Proof. Let $\psi_{\ell}$ is soft closed mapping. Let $v \in \mathcal{J}$ and let $\mathcal{F}_{R_{v}}$ be a closed soft set of $\left(\mathcal{M}_{v}, \tilde{u}_{v}, R_{v}\right)$. Then, by Theorem 4.2, $\mathcal{F}_{R_{v}} \times \underset{\substack{\alpha \neq \mathcal{J} \\ \alpha \in \mathcal{J}}}{\alpha}$ is a closed soft set of $\left(\prod_{\alpha \in \mathcal{J}} \mathcal{M}_{\alpha}, \otimes \tilde{u}, \prod_{\alpha \in \mathcal{J}} R_{\alpha}\right)$. Since $\psi_{\ell}$ is a soft closed mapping, then $\psi_{\ell}\left(\mathcal{F}_{R_{v}} \times \underset{\substack{\alpha \neq v \mathcal{M} \\ \alpha \in \mathcal{J}}}{\alpha}\right)$ is a closed soft set of $\left(\prod_{\alpha \in \mathcal{J}} \mathcal{N}_{\alpha}, \otimes \widetilde{\omega}, \prod_{\alpha \in \mathcal{J}} \mathcal{K}_{\alpha}\right)$. But $\psi_{\ell}\left(\mathcal{F}_{R_{v}} \times \underset{\substack{\alpha \neq v \\ \alpha \in \mathcal{J}}}{\widetilde{\alpha}} \mathcal{C}_{\alpha}\right)=\left(\psi_{\ell}\right)_{v}\left(\mathcal{F}_{R_{v}}\right) \times \underset{\substack{\alpha \neq v \\ \alpha \in \mathcal{J}}}{\alpha}$, hence $\psi_{\ell}\left(\mathcal{F}_{R_{v}}\right) \times \underset{\substack{\alpha \neq v \\ \alpha \in \mathcal{J}}}{\mathcal{N}_{\alpha}}$ is a closed soft set of $\left(\prod_{\alpha \in \mathcal{J}} \mathcal{N}_{\alpha}, \otimes \widetilde{\omega}, \prod_{\alpha \in \mathcal{J}} \mathcal{K}_{\alpha}\right)$. By Theorem $4.2, \psi_{\ell}\left(\mathcal{F}_{R_{v}}\right)$ is a closed soft set of $\left(\mathcal{N}_{v}, \widetilde{\omega}_{v}, \mathcal{K}_{v}\right)$. Hence, $\left(\psi_{\ell}\right)_{v}$ is soft closed mapping for each $\alpha \in \mathcal{J}$.

Conversely, let $\left(\psi_{\ell}\right)_{\alpha}$ be a soft closed mapping for each $\alpha \in \mathcal{J}$. Now, we shall prove that $\psi_{\ell}$ is a soft closed mapping. Suppose that $\psi_{\ell}$ is not a soft closed mapping. Then, there exists a closed soft set $\mathcal{F}_{\prod_{\alpha \in \mathcal{J}} R_{\alpha}}$ of $\left(\prod_{\alpha \in \mathcal{J}} \mathcal{M}_{\alpha}, \otimes \tilde{u}, \prod_{\alpha \in \mathcal{J}} R_{\alpha}\right)$ such that $\psi_{\ell}\left(\mathcal{F}_{\prod_{\alpha \in \mathcal{J}} R_{\alpha}}\right)$ is not closed soft set of $\left(\prod_{\alpha \in \mathcal{J}} \mathcal{N}_{\alpha}, \otimes \widetilde{\omega}, \prod_{\alpha \in \mathcal{J}} \mathcal{K}_{\alpha}\right)$. This implies that $\otimes \widetilde{\omega}\left(\psi_{\ell}\left(\mathcal{F}_{\prod_{\alpha \in \mathcal{J}} R_{\alpha}}\right)\right) \nsubseteq \psi_{\ell}\left(\mathcal{F}_{\prod_{\alpha \in \mathcal{J}} R_{\alpha}}\right)$. From the definition of $\otimes \widetilde{\omega}$, we get that $\left.\prod_{\alpha \in \mathcal{J}} \widetilde{\omega}_{\alpha}\left(\left(p_{\mathcal{N}_{\alpha}}, q_{\mathcal{K}_{\alpha}}\right)\left(\psi_{\ell}\left(\mathcal{F}_{\prod_{\alpha \in \mathcal{J}} R_{\alpha}}\right)\right)\right)\right) \nsubseteq \psi_{\ell}\left(\mathcal{F}_{\prod_{\alpha \in J} R_{\alpha}}\right)$, which implies that there exists $v \in \mathcal{J}$ such that $\widetilde{\omega}_{v}\left(p_{\mathcal{N}_{v}}, q_{\mathcal{K}_{v}}\right)\left(\psi_{\ell}\left(\mathcal{F}_{\prod_{\alpha \in \mathcal{J}} R_{\alpha}}\right) \sqsubseteq\left(p_{\mathcal{N}_{v}}, q_{\mathcal{K}_{v}}\right)\left(\psi_{\ell}\left(\mathcal{F}_{\prod_{\alpha \in \mathcal{J}} R_{\alpha}}\right)\right.\right.$. Therefore, $\widetilde{\omega}_{v}\left(\left(\psi_{\ell}\right)_{v}\left(\left(p_{\mathcal{M}_{v}}, q_{R_{v}}\right)\left(\mathcal{F}_{\prod_{\alpha \in \mathcal{J}} R_{\alpha}}\right)\right) \nsubseteq\left(\psi_{\ell}\right)_{v}\left(p_{\mathcal{M}_{v}}, q_{R_{v}}\right)\left(\mathcal{F}_{\prod_{\alpha \in J} R_{\alpha}}\right)\right.$.

Theorem 4.6. Let $(\mathcal{N}, \widetilde{\omega}, \mathcal{K})$ be a soft-cs, $\left\{\left(\mathcal{M}_{\alpha}, \tilde{u}_{\alpha}, R_{\alpha}\right): \alpha \in \mathcal{J}\right\}$ be a family of soft-cs's, and $\psi_{\ell}:(\mathcal{N}, \widetilde{\omega}, \mathcal{K}) \rightarrow\left(\prod_{\alpha \in \mathcal{J}} \mathcal{M}_{\alpha}, \otimes \tilde{u}, \prod_{\alpha \in \mathcal{J}} R_{\alpha}\right)$ is a soft mapping. Then, $\psi_{\ell}$ is soft continuous if and only if $\left(p_{\mathcal{M}_{\alpha}}, q_{R_{\alpha}}\right) o \psi_{\ell}$ is soft continuous for each $\alpha \in \mathcal{J}$.

Proof. Let $\psi_{\ell}$ be soft continuous. From Theorem 4.1, $\left(p_{\mathcal{M}_{\alpha}}, q_{R_{\alpha}}\right)$ is soft continuous for each $\alpha \in \mathcal{J}$ and by Proposition 3.9, $\left(p_{\mathcal{M}_{\alpha}}, q_{R_{\alpha}}\right) o \psi_{\ell}$ is soft continuous for each $\alpha \in \mathcal{J}$.

Conversely, let $\left(p_{\mathcal{M}_{\alpha}}, q_{R_{\alpha}}\right) o \psi_{\ell}$ be soft continuous for each $\alpha \in \mathcal{J}$. We must prove that $\psi_{\ell}$ is soft continuous. Suppose that $\psi_{\ell}$ is not soft continuous. Then, there exists a soft set $H_{\mathcal{K}} \in \mathcal{S} \mathcal{S}(\mathcal{N}, \mathcal{K})$ such that $\psi_{\ell}\left(\widetilde{\omega}\left(H_{\mathcal{K}}\right)\right) \nsubseteq \otimes \tilde{u}\left(\psi_{\ell}\left(H_{\mathcal{K}}\right)\right)$, which implies that $\psi_{\ell}\left(\widetilde{\omega}\left(H_{\mathcal{K}}\right)\right) \nsubseteq \prod_{\alpha \in \mathcal{J}}\left(\tilde{u}_{\alpha}\left(\left(p_{\mathcal{M}_{\alpha}}, q_{R_{\alpha}}\right)\left(\psi_{\ell}\left(H_{\mathcal{K}}\right)\right)\right.\right.$. Then, there exists $v \in \mathcal{J}$ such that $\left(p_{\mathcal{M}_{v}}, q_{R_{v}}\right)\left(\psi_{\ell}\left(\widetilde{\omega}\left(H_{\mathcal{K}}\right)\right) \nsubseteq \tilde{u}_{v}\left(\left(p_{\mathcal{M}_{v}}, q_{R_{v}}\right)\left(\psi_{\ell}\left(H_{\mathcal{K}}\right)\right)\right.\right.$. Since, for all $\alpha \in \mathcal{J}, \quad\left(p_{\mathcal{M}_{\alpha}}, q_{R_{\alpha}}\right) o \psi_{\ell} \quad$ is $\quad$ soft $\quad$ continuous, then $\left.\left.\left(\left(p_{\mathcal{M}_{v}}, q_{R_{v}}\right) o \psi_{\ell}\right)\left(\widetilde{\omega}\left(H_{\mathcal{K}}\right)\right)\right) \sqsubseteq \tilde{u}_{v}\left(\left(p_{\mathcal{M}_{v}}, q_{R_{v}}\right) o \psi_{\ell}\right)\left(H_{\mathcal{K}}\right)\right) . \quad$ Then, $\quad\left(p_{\mathcal{M}_{v}}, q_{R_{v}}\right)\left(\psi_{\ell}\left(\widetilde{\omega}\left(H_{\mathcal{K}}\right)\right)\right.$ 드 $\tilde{u}_{v}\left(\left(p_{\mathcal{M}_{v}}, q_{R_{v}}\right)\left(\psi_{\ell}\left(H_{\mathcal{K}}\right)\right)\right.$, which is a contraction. Consequently, $\psi_{\ell}$ is soft continuous mapping. 
Theorem 4.7 Let $\left\{\left(\mathcal{M}_{\alpha}, \tilde{u}_{\alpha}, R_{\alpha}\right): \alpha \in \mathcal{J}\right\}$ and $\left\{\left(\mathcal{N}_{\alpha}, \widetilde{\omega}_{\alpha}, \mathcal{K}_{\alpha}\right): \alpha \in \mathcal{J}\right\}$ be families of soft-cs's. For each $\alpha \in \mathcal{J}$, let $\left(\psi_{\ell}\right)_{\alpha}:\left(\mathcal{M}_{\alpha}, \tilde{u}_{\alpha}, R_{\alpha}\right) \rightarrow\left(\mathcal{N}_{\alpha}, \widetilde{\omega}_{\alpha}, \mathcal{K}_{\alpha}\right)$ be a soft mapping and let $\psi_{\ell}:\left(\prod_{\alpha \in \mathcal{J}} \mathcal{M}_{\alpha}, \otimes \tilde{u}, \prod_{\alpha \in \mathcal{J}} R_{\alpha}\right) \rightarrow\left(\prod_{\alpha \in \mathcal{J}} \mathcal{N}_{\alpha}, \otimes \widetilde{\omega}, \prod_{\alpha \in \mathcal{J}} \mathcal{K}_{\alpha}\right), \quad$ defined $\quad$ by $\quad \psi_{\ell}\left(\left(x_{\alpha_{\gamma_{\alpha}}}\right)_{\alpha \in \mathcal{J}}\right)=$ $\left(\left(\psi_{\ell}\right)_{\alpha}\left(x_{\alpha_{r_{\alpha}}}\right)\right)_{\alpha \in \mathcal{J}}$. Then, $\psi_{\ell}$ is soft continuous if and only if $\left(\psi_{\ell}\right)_{\alpha}$ is soft continuous for each $\alpha \in \mathcal{J}$.

Proof. Let $\psi_{\ell}$ be a soft continuous, let $v \in \mathcal{J}$, and let $H_{R_{v}} \subseteq \widetilde{\mathcal{M}}_{v}$. Then,

$$
\begin{aligned}
& \left(\psi_{\ell}\right)_{v}\left(\tilde{u}_{v}\left(H_{R_{v}}\right)\right)=\left(p_{\mathcal{M}_{v}}, q_{R_{v}}\right)\left(\left(\psi_{\ell}\right)_{v}\left(\tilde{u}_{v}\left(H_{R_{v}}\right)\right) \times \prod_{\substack{\alpha \neq v \\
\alpha \in \mathcal{J}}}\left(\psi_{\ell}\right)_{\alpha}\left(\tilde{u}_{\alpha}\left(\widetilde{\mathcal{M}}_{\alpha}\right)\right)\right. \\
& =\left(p_{\mathcal{M}_{v}}, q_{R_{v}}\right)\left(\psi _ { \ell } \left(\left(\tilde{u}_{v}\left(H_{R_{v}}\right)\right) \times \prod_{\substack{\alpha \neq v \\
\alpha \in \mathcal{J}}}\left(\tilde{u}_{\alpha}\left(\widetilde{\mathcal{M}}_{\alpha}\right)\right)\right.\right. \\
& =\left(p_{\mathcal{M}_{v}}, q_{R_{v}}\right)\left(\psi_{\ell}\left(\otimes \tilde{u}\left(H_{R_{v}} \times \underset{\substack{\alpha \neq v \mathcal{M} \\
\alpha \in \mathcal{J}}}{\underset{\alpha \in \mathcal{J}}{\alpha}}\right)\right)\right. \\
& \text { 드 }\left(p_{\mathcal{M}_{v}}, q_{R_{v}}\right)\left(\otimes \widetilde { \omega } ( \psi _ { \ell } ( H _ { R _ { v } } \times \underset { \substack { \alpha \neq v \mathcal { M } \\
\alpha \in \mathcal { J } } } { \underset { \in \in J } { \alpha } } ) ) \left(\psi_{\ell}\right.\right. \text { is soft continuous) } \\
& =\left(p_{\mathcal{M}_{v}}, q_{R_{v}}\right)\left(\otimes \widetilde{\omega}\left(\left(\psi_{\ell}\right)_{v}\left(H_{R_{v}}\right)\right) \times \prod_{\alpha \in J}^{\alpha \neq v}\left(\psi_{\ell}\right)_{\alpha}\left(\widetilde{\mathcal{M}}_{\alpha}\right)\right) \\
& =\left(p_{\mathcal{M}_{v}}, q_{R_{v}}\right)\left(\widetilde{\omega}_{v}\left(\left(\psi_{\ell}\right)_{v}\left(H_{R_{v}}\right)\right) \times \prod_{\substack{\alpha \neq v \\
\alpha \in \mathcal{J}}} \widetilde{\omega}_{\alpha}\left(\left(\left(\psi_{\ell}\right)_{\alpha}\left(\widetilde{\mathcal{M}}_{\alpha}\right)\right)\right.\right. \\
& =\widetilde{\omega}_{v}\left(\left(\psi_{\ell}\right)_{v}\left(H_{R_{v}}\right)\right. \text {. }
\end{aligned}
$$

Thus, $\left(\psi_{\ell}\right)_{v}\left(\tilde{u}_{v}\left(H_{R_{v}}\right)\right) \sqsubseteq \widetilde{\omega}_{v}\left(\left(\psi_{\ell}\right)_{v}\left(H_{R_{v}}\right)\right.$. Consequently, $\left(\psi_{\ell}\right)_{v}$ is soft continuous for all $v \in \mathcal{J}$. Conversely, let $\left(\psi_{\ell}\right)_{\alpha}$ be soft continuous for each $\alpha \in \mathcal{J}$ and let $H_{\prod_{\alpha \in \mathcal{J} R_{\alpha}} \subseteq \prod_{\alpha \in \mathcal{J}} \mathcal{M}}^{\alpha}$. Then,

$$
\begin{aligned}
& \psi_{\ell}\left(\otimes \tilde{u}\left(H_{\prod_{\alpha \in \mathcal{J}} R_{\alpha}}\right)\right)=\psi_{\ell}\left(\prod_{\alpha \in \mathcal{J}} \tilde{u}_{\alpha}\left(\left(p_{\mathcal{M}_{\alpha}}, q_{R_{\alpha}}\right)\left(H_{\prod_{\alpha \in \mathcal{J}} R_{\alpha}}\right)\right)\right. \\
& \quad=\prod_{\alpha \in \mathcal{J}}\left(\psi_{\ell}\right)_{\alpha}\left(\prod_{\alpha \in \mathcal{J}} \tilde{u}_{\alpha}\left(\left(p_{\mathcal{M}_{\alpha}}, q_{R_{\alpha}}\right)\left(H_{\prod_{\alpha \in \mathcal{J}} R_{\alpha}}\right)\right)\right. \\
&=\prod_{\alpha \in \mathcal{J}}\left(\psi_{\ell}\right)_{\alpha}\left(\tilde{u}_{\alpha}\left(\left(p_{\mathcal{M}_{\alpha}}, q_{R_{\alpha}}\right)\left(H_{\prod_{\alpha \in \mathcal{J}} R_{\alpha}}\right)\right)\right. \\
& \sqsubseteq \prod_{\alpha \in \mathcal{J}} \widetilde{\omega}_{\alpha}\left(\left(\psi_{\ell}\right)_{\alpha}\left(\left(p_{\mathcal{M}_{\alpha}}, q_{R_{\alpha}}\right)\left(H_{\prod_{\alpha \in \mathcal{J}} R_{\alpha}}\right)\right)\right. \\
&=\prod_{\alpha \in \mathcal{J}} \widetilde{\omega}_{\alpha}\left(\left(p_{\mathcal{M}_{\alpha}}, q_{R_{\alpha}}\right)\left(\left(\psi_{\ell}\left(H_{\prod_{\alpha \in \mathcal{J}} R_{\alpha}}\right)\right)\right)\right. \\
&=\otimes \widetilde{\omega}\left(\psi_{\ell}\left(H_{\prod_{\alpha \in \mathcal{J}} R_{\alpha}}\right)\right.
\end{aligned}
$$

Therefore, $\psi_{\ell}$ is soft continuous.

\section{Conclusions}

Soft closure spaces are a very new concept and an important topic for investigators because it is more general as compared to the concept of soft topological spaces. The notions of soft continuous and soft closed (resp. open) mappings were introduced in this paper, and some related properties and theorems were developed. To explain our notions, we put forward some examples.

\section{References}

1. Molodtsov, D. 1999. Soft set theory-First results. Comput. Math. Appl., 37:19-31.

2. Aktas, H. and Cagman, N. 2007. Soft sets and soft groups. Information Sci., 177:2726-2735.

3. Shabir, M. and Naz, M. 2011. On soft topological spaces. Computers and Mathematics with Applications, 61:1786-1799.

4. Gowri, R. and Jegadeesan, G. 2014. On soft Čech closure spaces. International Journal of Mathematics Trends and Technology, 9(2):122-127.

5. Krishnaveni, J. and Sekar, C. 2014. C ech Soft Closure Spaces. International Journal of Mathematical Trends and Technology, 6:123-135.

6. Majeed, R. N. 2018. Čech fuzzy soft closure spaces. International Journal of Fuzzy System Applications, 7(2):62-74.

7. Majeed, R. N. and Maibed, L. H. 2019. Lower separeation axioms in $\check{C}$ ech fuzzy soft closure spaces. Gazi University Journal of Science, 32(4): 1254-1269. DOI: 10.35378/gujs.433174.

8. Majeed, R. N. 2019. Generalized closed fuzzy soft sets in Čech fuzzy soft closure spaces. Journal of Interdisciplinary Mathematics, 22(8): 1433-1441. DOI: 10.1080/09720502.2019.1700931.

9. Ekram, S. T. and Majeed, R. N. 2020. Soft closure spaces. IOP Conf. Series: Journal of Physics: Conference Series, 1591:012076.

10. Čech, E. 1968. Topological Spaces. Topological Papers of Eduard Čech, Academia, Prague, 436472. 
11. Kharal, A. and Ahmad, B. 2011. Mappings on Soft Classes. New Math. Nat. Comput., 7 (3):471481.

12. Majumdar, P. and Samanta, S.K. 2010. On soft mappings. Computers and Mathematics with Applications, 60:2666-2672.

13. Boonpok, C. 2009. On continuous maps in closure spaces. General Mathematics, 17(2):127-134.

14. Maji, P. K., Biswas, R. and Roy, R. 2003. Soft set theory. Comput. Math. Appl., 45:555-562.

15. Feng, F, Jun, Y. B., and Zhao, X. 2008. Soft semirings. Comput. Math. Appl., 56:2621-2628.

16. Das, S. and Samanta, S. K. 2013. soft metric. Annals of Fuzzy Mathematics and Informatics, 1:7794.

17. Aygünoğlu, A and Aygün, H. 2012.Some notes on soft topological spaces. Neural Comput and Applications. 21(2012): 113-119. DOI 10.1007/ s00521-011-0722-3.

18. Babitha, K. V. and Sunil, J. J. 2010. Soft set relations and functions. Comput. Math. Appl., 60: 1840-1849. 